\title{
La captación de inversiones por el sistema financiero de Chimborazo, análisis desde la perspectiva del cliente
}

\section{The capture of investments by the financial system of Chimborazo, analysis from the client's perspective}

Denise Liliana Pazmiño Garzón. 1, Yolanda Patricia Moncayo Sánchez. ${ }^{2}$, Angelita Genoveva Tapia Bonifaz. ${ }^{3}$ \& Ginno Sidney Jarrin Zambrano. ${ }^{4}$

\begin{abstract}
DOI: https://doi.org/10.33262/cienciadigital.v3i3.1.708

This research aimed to determine the amount of fundraising by the Chimborazo financial system in 2018, and at the same time determine the degree of satisfaction and the variables that the client considers investing in a financial entity. This study was based on a qualitative and quantitative approach, with a deductive process, the scope was descriptive since a reality is detailed, we worked with a sample of 384 clients of the Chimborazo financial system, the analytical and synthetic methods were combined. Among the results obtained, it was determined that the province's financial system raised $\$ 791,328,665.77$ dollars, of which $64.26 \%$ received cooperatives, $34.29 \%$ banks, and $1.44 \%$ mutualists ; In the same way, it was identified that only $31 \%$ of the clients are totally satisfied with the service offered by these entities, $23 \%$ are not very satisfied, and $46 \%$ are not at all satisfied, on the other hand $46 \%$ of the clients To make the investment decision in a financial institution, they first consider security, $36 \%$ interest rates, $11 \%$ liquidity and $7 \%$ risk. Based on the foregoing, it is concluded that
\end{abstract}

\footnotetext{
${ }^{1}$ Escuela Superior Politécnica de Chimborazo, Facultad de Administración de Empresas, Riobamba, Ecuador, denise.pazmiño@espoch.edu.ec

${ }^{2}$ Escuela Superior Politécnica de Chimborazo, Facultad de Administración de Empresas, Riobamba, Ecuador, yolanda.moncayo@espoch.edu.ec

${ }^{3}$ Escuela Superior Politécnica de Chimborazo, Facultad de Administración de Empresas, Riobamba, Ecuador, genoveva.tapia@espoch.edu.ec

${ }^{4}$ Escuela Superior Politécnica de Chimborazo, Facultad de Administración de Empresas, Riobamba, Ecuador, sidney.jarrin@espoch.edu.ec
} 
cooperatives are the financial entities with the highest market share in the province of Chimborazo, that the majority of their clients are not very satisfied and not satisfied with the services they receive, and that the main variables analyzed the client to invest in a financial entity is security and interest rates.

Keywords: Investment, savings, catchment, financial system, client.

\section{RESUMEN}

Esta investigación tuvo como objetivo determinar el monto de las captaciones por parte del sistema financiero de Chimborazo en el año 2018, y a la vez determinar el grado de satisfacción y las variables que considera el cliente para invertir en una entidad financiera. Este estudio se fundamentó en un enfoque cualitativo y cuantitativo, con un proceso deductivo, el alcance fue descriptivo ya que se detalla una realidad, se trabajó con una muestra de 384 clientes del sistema financiero de Chimborazo, se combinaron los métodos analítico y sintético. Entre los resultados obtenidos, se determinó que el sistema financiero de la provincia capto $\$ 7913328.665,77$ dólares, de los cuales el 64,26\% recibieron las cooperativas, el 34,29\% los bancos, y el 1,44\% las mutualistas; de igual manera se identificó, que tan solo el $31 \%$ de los clientes están totalmente satisfechos con el servicio que ofertan estas entidades, un $23 \%$ está poco satisfecho, y un $46 \%$ está nada satisfecho, por otro lado el $46 \%$ de los clientes para tomar la decisión de inversión en una institución financiera consideran en primer lugar la seguridad, un $36 \%$ las tasas de interés, el 11\% la liquidez y un 7\% el riesgo. Por lo expuesto, se concluye que las cooperativas son las entidades financieras que mayor participación de mercado tienen en la provincia de Chimborazo, que los clientes en su mayoría están poco satisfechos y nada satisfechos con los servicios que reciben, y que las principales variables que analiza el cliente para invertir en una entidad financiera es la seguridad y las tasas de interés.

Palabras claves: Inversión, ahorro, captación, sistema financiero, cliente.

\section{INTRODUCCIÓN}

En el Ecuador no es sencillo fomentar el ahorro y la inversión ya que las personas no tienen esta cultura debido a diversos factores tales como el reducido nivel de ingreso, el miedo generado por la incertidumbre económica, la falta de información, la apatía, escasez de empleo, entre otras razones.

También se debe tener presente que cada cliente es diferente, que no solo basta con obtener su ingreso dentro de la misma, sino también lograr una mayor analogía con él, logrando 
satisfacer sus deseos y expectativas a corto y largo plazo. No olvidemos que el éxito en toda organización depende de la permanencia de sus clientes.

El principal problema que se desarrolla en la provincia de Chimborazo es la falta de empleo, ya que como todos sabemos el empleo influye el desarrollo económico de la sociedad para incrementar su bienestar. Una contribución al problema también es la falta de ahorro y de conocimientos de las personas acerca de las inversiones disponibles, el miedo al uso de la tecnología, la falta de conocimiento que cada vez se implementan en las instituciones financieras

La administración de una institución financiera busca procesos más eficientes, con menos costos y que sea de impacto directo por ello se apega a una estrategia en que consiste en el crecimiento de los segmentos de la economía, con servicios y productos que satisfagan las necesidades de los clientes.

Actualmente en el país y de manera específica en el sector financiero ha sufrido una profunda transformación en la última década, factores como globalización, privatizaciones, entrada de nuevos competidores, el desarrollo y aplicación de la Tecnología de la Información y conocimiento, esto ha unido a una nueva cultura financiera de los ciudadanos.

Se ha logrado un fuerte incremento de competencia, lo que ha conllevado a que las entidades financieras se preocupen en lograr cambios significativos en su gestión, para que de una u otra manera lograr la fidelidad de los clientes que está marcada por variables bien definidas en cuanto a la preferencia de un banco, cooperativa, mutualista, sociedad financiera.

El objetivo de esta investigación es determinar el volumen de captaciones por parte del sistema financiero de Chimborazo en el año 2018, y a la vez determinar el grado de satisfacción y las variables que considera el cliente para invertir en una institución financiera, con el fin de establecer estrategias comerciales para mejorar la captación de inversiones y la fidelización de los clientes del sistema financiero de esta provincia.

\section{Desarrollo}

\section{Marco Teórico Referencial}

\section{Captación y colocación}

De acuerdo con la (Subgerencia Cultural del Banco de la República , 2015), la captación es la recolección de dinero de diferentes personas u organizaciones dependiendo del tipo de cuenta que tengan los mismos entre los que pueden ser: cuenta de ahorros, cuenta corriente, certificados de depósitos a término fijo entre otros: los mismos que ganan un porcentaje de interes determinada por la institución bancaria. 
Dentro del proceso de captación se encuentran los siguientes actores:

a. Los accionistas.

b. Los depositantes.

c. El depósito.

- Cuenta corriente.

- Cuenta de ahorros.

- Depósitos a plazo.

- Pólizas de acumulación.

- Contratos de reporto.

- Depósitos en garantía.

La colocación es la "negociación destinada a la venta de una nueva emisión de valores, o a la concesión de un préstamo a largo plazo" (Bancafácil, 2006).

La colocación permite poner dinero en circulación en la economía, ya que los bancos toman el dinero o los recursos que obtienen a través de la captación y, con éstos, otorgan créditos a las personas, empresas u organizaciones que los soliciten. Por dar estos préstamos los bancos cobran, dependiendo del tipo de préstamo, una cantidad determinada de dinero llamada intereses (intereses de colocación), la cual se define a través de la tasa de interés de colocación (Subgerencia Cultural del Banco de la República, 2015).

Dentro de las principales inversiones que pueden generarse a través del proceso de colocación son:

a. Compra de activos.

b. Colocación de créditos en cartera.

- Préstamos con garantía personal, quirografarios o de consumo.

- Documentos descontados.

- Sobregiros ocasionales.

- Contrato de crédito a cuenta corriente.

- Préstamos prendarios.

- Préstamos hipotecarios

\section{Inversión}

Según Amling, la inversión puede ser definida como la compra de cualquier activo real o financiero, que ofrece una utilidad en forma de capital, ganancia, interés o dividendo. 
Asimismo, la inversión se conoce como el sacrificio que se hace de dinero actual para obtener más en el futuro. La inversión generalmente incluye dos elementos: tiempo y riesgo. El sacrificio es tomado en el presente y es real, mientras que la ganancia se genera en el futuro y es generalmente incierta. (Alexander, Sharpe, \& Bailey, 2003)

(Hernández, Hernández, \& Hernández, 2005) dice que la "inversión es el conjunto de recursos que se emplean para producir un bien o servicio y generar una utilidad". (p. 3). También a la inversión se le puede considerar como el recurso monetario que se emplea con la finalidad de obtener un beneficio mayor a la inversión empleada por lo que podemos definir a la inversión como el capital monetario empleado para satisfacer las necesidades del ser humano mediante la búsqueda de un lucro.

\section{Inversión real}

La inversión real es la que se hace en bienes tangibles como planta y equipo, inventarios, terrenos o bienes raíces. En el caso de una persona moral, son las inversiones que se hacen para asegurar la operación normal de un negocio.

Asimismo, en el caso de una persona física, por lo regular la inversión se realiza con el objeto de asegurar su vivienda, es decir, una inversión en bienes raíces. (Heyman, 2001)

\section{Inversión y financiera}

Las inversiones financieras tienen la característica de ser líquidas o de fácil realización y el inversionista puede deshacerse de ellas en el momento en que lo necesite para el negocio.

\section{Inversión directa e indirecta}

Se considera inversión directa, aquella en la que el inversionista adquiere directamente un derecho sobre un valor o propiedad, por ejemplo cuando una persona compra una acción, para conservar el valor de sus recursos u obtener de ellos un beneficio.

Se considera como inversión indirecta, la que se realiza en una cartera o grupo de valores o propiedades. Por ejemplo, cuando un inversionista compra una acción de un fondo mutualista, el cual es una cartera diversificada de valores emitidos por varias empresas, está realizando una inversión directa en el fondo mutualista, pero a su vez, realiza una inversión indirecta en las empresas emisoras de valores, por lo tanto, será dueño de un derecho sobre una fracción de la cartera y no sobre los valores de las empresas. (Gitman \& Joehnk, 2009)

\section{Ahorro}


Al igual que la inversión, es un determinante fundamental del crecimiento del Producto Interno Bruto (PIB) a largo plazo y de los niveles de vida futuros, ya que se transforma en inversión y ésta equivale a consumo diferido y ampliado.

\section{Ahorro Interno y Externo}

Samuelson et al (1999:150) considera que el ahorro interno o doméstico de un país se compone por el ahorro personal, el ahorro de las empresas (el ahorro personal y el ahorro de las empresas conforman al ahorro privado) y el ahorro del sector público (este tipo de ahorro se genera con finanzas públicas sanas, en otras palabras, con un superávit fiscal).

El ahorro externo por su parte se compone por la inversión extranjera directa, los prestamos recibidos por el sector público y privado no monetario, el cambio en las reservas internacionales, las transferencias de capital y los errores u omisiones. Por último, el ahorro financiero se define como la diferencia entre el agregado monetario más amplio (M4) y los billetes y monedas en poder del público.

\section{Los determinantes del ahorro}

Cuando se habla de los determinantes del ahorro, ya sea a nivel macroeconómico o a nivel de hogares, se refiere a aquellas variables que tienen incidencia sobre las decisiones de consumo y ahorro de las familias. Sin embargo, su efecto sobre el consumo va depender de las expectativas acerca del impacto permanente o transitorio de ellas.

Algunas variables que son determinantes del ahorro a nivel macroeconómico son el tipo de cambio real, importaciones de bienes de consumo durable y no durable y el ahorro externo. Y algunas variables internas como la disponibilidad de crédito, inflación, impuestos, ingresos laborales, educación, remesas, edad, etc.

\section{Tasa de interés}

Premio o precio que se recibe a cambio de ceder la disponibilidad de dinero durante un tiempo determinado. Al ser pagadero también en dinero, se expresa en forma de una proporción, generalmente en forma porcentual.

\section{Tasa de rendimiento}

Beneficio que produce una inversión expresado como un porcentaje del monto invertido. Tasa que expresa el rendimiento de una inversión. 


\section{Tasa efectiva}

Tasa que efectivamente se recibe, o se paga según sea el caso, por el periodo de tiempo en que se toma prestado o se invierte el dinero.

\section{Tasa pasiva}

Tasa que un Banco paga a sus depositantes; en general es la lasa que un intermediario financiero lleva a cabo la captación de recursos.

\section{Las tasas de interés y las decisiones de los inversionistas}

Según (Oppers, 2011) Se observa que los factores que sí consideran al tomar sus decisiones son las perspectivas favorables y estables de crecimiento, un bajo riesgo país incluidas la estabilidad política y económica y un tipo de cambio estable. Todo esto tiene sentido para los inversionistas a largo plazo, como los fondos de pensiones y las compañías de seguros.

Estos inversionistas suelen tener un horizonte de inversión a largo plazo, con obligaciones que con frecuencia se extienden a lo largo de décadas. Centran su atención en los rendimientos a largo plazo y - un factor importante - invierten su propio dinero, en lugar de invertir con dinero prestado.

\section{Las Expectativas Inflacionarias (riesgo inflación)}

La inflación reduce el poder adquisitivo del dinero. Cada aumento de un punto porcentual en la inflación representa aproximadamente una disminución de 1 por ciento de la cantidad de mercancías verdaderas y de servicios que se puedan comprar con un número dado de dólares en el futuro. Consecuentemente, los prestamistas, intentando proteger su poder adquisitivo, agregan la tasa de inflación prevista al tipo de interés que exigen. Los prestatarios están dispuestos a pagar esta tarifa más alta porque esperan que la inflación les permita compensar el préstamo con soles más baratos.

\section{La Devaluación Esperada}

Si se espera que suba el tipo de cambio las personas que pueden prestar reducirían su oferta de préstamos y los demandantes querrían endeudarse más generando un exceso de demanda por préstamos que elevaría la tasa de interés de equilibrio en moneda nacional. (Roca, 2002)

\section{Sistema financiero ecuatoriano.}


Un sistema financiero es un conjunto de instituciones que tiene como objetivo canalizar el ahorro de las personas. Esta canalización de recursos permite el desarrollo de la actividad económica (producir - consumir) haciendo que los fondos lleguen desde las personas que necesitan éstos recursos. (Robayo, 2012)

En nuestro país se encuentra compuesto por banco, sociedades financieras, cooperativas y mutualistas, instituciones financieras públicas, instituciones de servicios financieros, compañías de seguros y compañías auxiliares del sistema financiero, cada una de éstas realiza sus actividades dentro de la normativa vigente según su organismo de control, constituyéndose los bancos en el mayor y más importante participante del mercado con más del $90 \%$ de las operaciones del total del sistema. (Romero, 2019)

El sistema financiero es considerado como uno de los factores más importantes dentro de la economía, el mismo permite canalizar el ahorro hacia la inversión, por este motivo se ha convertido en el pilar fundamental para la generación de riqueza, razón por la cual, los entes económicos se han apoyado en las diversas instituciones financieras para la obtención de capital de trabajo, expansión productiva e infraestructura. (Romero, 2019)

Se puede entender al Sistema Financiero Nacional, como el conjunto de instituciones financieras (bancos, sociedades financieras, asociaciones mutualistas de ahorro y crédito para la vivienda y las cooperativas de ahorro y crédito) que realizan intermediación financiera con el público. (Romero, 2019)

\section{Ley general de Instituciones financieras}

El sistema financiero está conformado por el conjunto de Instituciones bancarias, financieras y demás empresas e Instituciones de derecho público o privado, debidamente autorizadas por la Superintendencia de Bancos y Seguros, que operan en la intermediación financiera. Es el conjunto de instituciones encargadas de la circulación del flujo monetario y cuya tarea principal es canalizar el dinero de los ahorristas hacia quienes desean hacer inversiones productivas. Sistema financiero del Ecuador, (2017), citado por (Román, 2018)

\section{Funcionamiento del Sistema Financiero.}

Las instituciones financieras reciben dinero de los depositantes y lo presta a quienes lo solicitan. Estas personas deben devolver el dinero con intereses, para que la institución pueda seguir prestando dinero. Este ciclo funciona de forma continua y simultánea. Las instituciones financieras son los intermediarios entre quienes tienen disponibilidad de recursos y quienes necesitan. A continuación, se presenta gráficamente cómo funciona el sistema financiero. (Romero, 2015) 


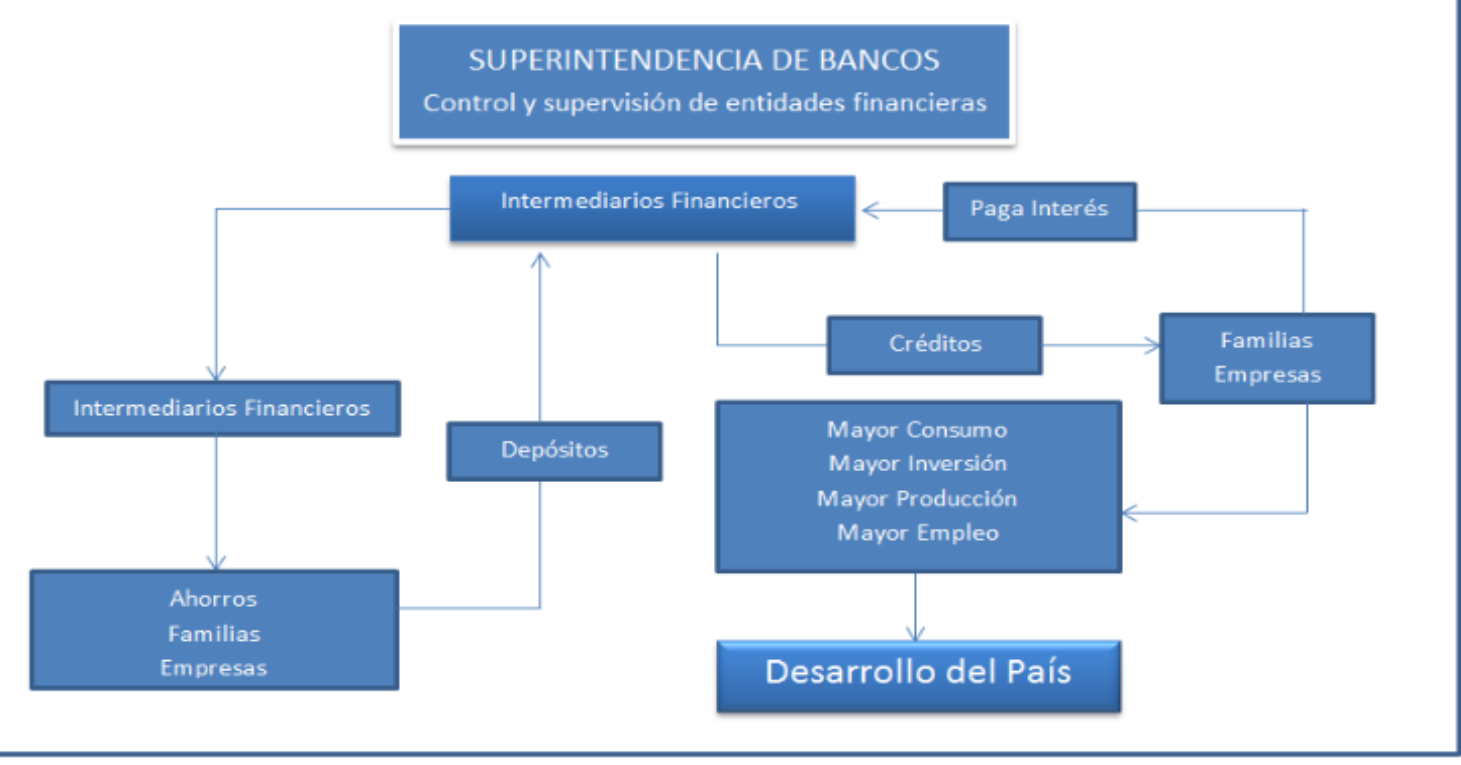

Figura 1: Funcionamiento del Sistema Financiero

Fuente: https://tusfinanzas.ec/category/sistema-financiero/

Elaborado por: Los autores

- La Superintendencia de Bancos es la institución encargada de controlar y supervisar a las instituciones financieras.

- Las familias y las empresas ahorran.

- Las familias depositan ese ahorro en una institución financiera.

- La institución les paga un interés, y a la vez les presta a otras familias y empresas.

- Las familias y empresas que tomaron créditos paga un interés.

- Esos recursos se vuelcan al consumo, a la inversión y a la actividad económica

- El aumento de la actividad económica hace crecer al país, y todos los ciudadanos se benefician. (Romero, 2015)

\section{¿Cómo se Regula el Sistema Financiero?}

La Junta de Política y Regulación Monetaria y Financiera es la responsable de la formación de políticas públicas, y la regulación y supervisión monetaria, crediticia, cambiaria, financiera, de seguros y valores. (Romero, 2015)

Los organismos de supervisión y control son la Superintendencia de Bancos (bancos, mutualistas y sociedades financieras), la Superintendencia de Economía Popular y Solidaria (cooperativas y mutualistas de ahorro y crédito de vivienda) y la Superintendencia de Compañías, Valores y Seguros (compañías de seguros). (Romero, 2015) 


\section{La Banca Pública}

Para propósitos nobles como erradicar la pobreza y fomentar la inversión y el ahorro en el Ecuador se creó la banca pública de desarrollo, la cual está conformada por el Banco del Estado (BEDE), el Banco Ecuatoriano de la Vivienda (BEV), el BanEcuador y la Corporación Financiera Nacional (CFN) y el Instituto Ecuatoriano de Créditos Educativos (IECE). Estas Instituciones se encuentran reguladas por la Superintendencia de Bancos; lo cual, causa un conflicto de intereses que imposibilita que la supervisión sea imparcial, pues el estado es supervisor y banquero a la vez.

Cabe indicar que el Banco del Estado (BEDE) trabaja con los gobiernos autónomos descentralizados; El Banco Ecuatoriano de la Vivienda (BEV) da crédito a los constructores; El IECE otorga créditos a los estudiantes para maestrías y posgrados; el BanEcuador ofrece microcrédito productivo y sobre todo crédito individual; la Corporación Financiera Nacional (CFN) tiene un segmento más corporativo y también se encarga de trabajar en las corresponsalías; es decir, de ser el "Eximbanco" (Exportaciones e importaciones de materias primas y líneas de capital) del Ecuador. (Calderón, 2006)

a) Productos y servicios

Este momento, todos están operando en primer piso en términos de otorgamiento de créditos; Banecuador está en los dos niveles, maneja cuentas corrientes y de ahorro. La CFN también hace operaciones de ambos tipos y entraría en una fase de tener cuentas corrientes y de ahorro. El BEDE sólo entrega crédito; el IECE otorga créditos directos y se está pensando cómo diversificar sus productos. El BEV actúa como entidad de primer piso y tiene algo de atención a los ciudadanos con cuentas de ahorro.

La banca pública de desarrollo de segundo piso ayuda a salvaguardar la liquidez de la banca de primer piso y constituye una forma más barata de obtener recursos económicos para las entidades de primer piso y su giro de negocio. También ayuda a promover mejores plazos para los créditos, mayores montos y menores tasas de interés, beneficiando directamente a los beneficiarios finales de los créditos, y así de esta manera ayuda a incrementar la profundización financiera. (Aguirre \& Andrango, 2011)

\section{Cooperativismo}

"La cooperativa es una sociedad de derecho privado, formada por personas naturales o jurídicas que sin perseguir finalidades de lucro, tienen por objeto planificar y realizar actividades o trabajos de beneficio social o colectivo a través de una empresa manejada en común y formado con la aportación económica, intelectual y moral de sus miembros" (Caraballo, 2011) 
El mercado financiero ecuatoriano está compuesto por 40 cooperativas de ahorro y crédito, entre las principales: 29 de Octubre, Andalucía y Alianza del Valle, etc. Las cooperativas, se clasifican según la actividad que vayan a desarrollar como cooperativas de producción, consumo, crédito y servicios; y por la capacidad que tengan como cooperativas abiertas (captan dinero del público en general) y cooperativas cerradas (agrupan a socios que pertenecen a un solo grupo del trabajo). (Recalde, 2010)

b) Productos y servicios

- Los ahorros. La cooperativa tiene como finalidad fundamental la de ofrecer a sus afiliados el servicio de préstamos; ahora, para contar con las disponibilidades monetarias que le permita ofrecer el servicio tiene que formar primero un capital accionario o de operación a base de los depósitos económicos efectuados por todos los miembros de la sociedad. (Superintendencia de Bancos, 2010)

- Depósitos a plazo fijo. Los socios de una Cooperativa de Ahorro y Crédito, a más de depositar dinero en las cuentas de Certificados y Ahorros, pueden hacerlo también en otra que sea denominada depósitos a plazo fijo. Este dinero gana un interés convenido de acuerdo al plazo que dura el depósito, cuyas tasas constan en el Reglamento respectivo de la cooperativa.

- Los préstamos. Constituyen el principal y más significativo de los servicios. Es un derecho que utiliza el socio como reconocimiento al mérito de la cooperación. Es un acto de solidaridad y confianza, los miembros de la sociedad se prestan dinero y se garantizan mutuamente, bajo normas justas. (Recalde, 2010)

\section{El Mutualismo}

Constituye la asociación libre, sin fines de lucro, por personas inspiradas en la solidaridad, con el objeto de brindarse ayuda reciproca frente a riesgos eventuales o de concurrir a su bienestar material y espiritual mediante una contribución periódica. (Avila, 2011)

c) Productos y servicios

Entre los principales productos tenemos:

- Ahorro tradicional. Es la cuenta de ahorros que permite hacer rendir el dinero del socio de una manera segura.

- Ahorro programado. Aquí el socio empieza a construir sus sueños, ya que mediante el ahorro planificado podrá ir acumulando su dinero. 
- Cuenta constructiva. Este producto está diseñado para ayudar a que los asociados conviertan sus sueños en realidad. Consta de dos partes: Ahorro Tradicional y Ahorro Planificado.

- Inversiones. Con este producto, el socio podrá invertir su dinero a través de certificados de inversión de acuerdo a planes a corto, mediano y largo plazo. Crédito hipotecario. - Es un crédito de amortización mensual pagadero a mediano o largo plazo, otorgado a personas naturales.

\section{Banca Privada}

Los Bancos "Son entidades de origen privado y público autorizadas y constituidas legalmente para captar dinero del público en moneda nacional o extranjera en forma habitual o continua; conceder créditos para estimular el desarrollo económico social y productivo del país. Además, canalizar el ahorro y la inversión" (Recalde, 2010)

El mercado financiero ecuatoriano está compuesto por 22 entidades privadas y públicas, nacionales y extranjeras. (Recalde, 2010)

Las actividades bancarias están determinadas básicamente por el funcionamiento de los bancos públicos y privados, quienes tienen una amplia gama de operaciones que permiten el desarrollo de importantes sectores de la producción, en especial por el crédito, sustancial característica de estas actividades. (Recalde, 2010)

Los bancos públicos tienen como característica, otorgar créditos que estimulen el desarrollo económico y social; por lo tanto, las condiciones de los préstamos tienen sus ventajas con respecto de los bancos privados, sea por el plazo, tasas de interés, años de gracia, garantías y formas de pago. Luis Chiriboga, (2007), citado por (Aguirre \& Andrango, 2011)

Los bancos privados en cambio si bien apoyan el desarrollo productivo de un país, persiguen el beneficio, por ello las condiciones de los préstamos son diferentes con relación a los bancos públicos. (Recalde, 2010)

d) Productos y servicios financieros

De acuerdo con el Art. 51 de la ley de instituciones del Sistema Financiero. "Los Bancos podrán efectuar las siguientes operaciones en moneda nacional o extranjera, o en unidades de cuenta establecida en la ley. A continuación, mencionamos algunos:

- Recibir recursos del público en depósitos a la vista.

- Recibir a depósitos a Plazo. 
- Asumir obligaciones por cuenta de terceros.

- Emitir obligaciones y cédulas garantizadas con sus activos y patrimonio.

- Recibir préstamos y aceptar créditos. De Instituciones Financieras del país y del exterior.

- Otorgar préstamos hipotecarios y prendarios, con o sin emisión de títulos; así como préstamos quirografarios.

\section{Perspectiva del Cliente:}

Para Kaplan y Norton (2.002), en la perspectiva del cliente del Cuadro de Mando Integral (CMI), las empresas identifican los segmentos de cliente y de mercado en que han elegido competir. Estos segmentos representan las fuentes que proporcionarán el componente de ingresos de los objetivos financieros del ente financiero. Además, esta perspectiva permite que las instituciones financieras equiparen sus indicadores claves sobre clientes (satisfacción, fidelidad, retención, adquisición y rentabilidad) con los segmentos de clientes y mercado seleccionados. También, permite identificar y medir de forma explícita las propuestas de valor añadido que entregarán a los segmentos de clientes y mercado seleccionados.

Por otra parte, los gerentes de la institución deben, dentro de la perspectiva de cliente, traducir las declaraciones de visión y estrategia en unos objetivos concretos basados en el mercado y los clientes. A su vez, deberán identificar los segmentos de mercado en sus poblaciones de clientes existentes y potenciales, y luego seleccionar los segmentos en los que eligen competir.

Se debe tener en cuenta que, generalmente, los clientes existentes y los potenciales no son homogéneos, y tienen preferencias diferentes y valoran de manera diferente los atributos del producto o servicio. Por ello, un proceso de formulación de estrategia debe revelar los diferentes segmentos de mercado o cliente y sus preferencias, en cuanto al precio, calidad, funcionalidad, imagen, prestigio, relaciones y servicio. Por tanto, la estrategia de la entidad bancaria puede definirse en función de esos segmentos de cliente y de mercado que elija convertir en objetivos; y el CMI debería identificar los objetivos del cliente de cada segmento seleccionado (Kaplan y Norton, 2.002).

Por otra parte, las propuestas de valor añadido a los clientes representan los atributos que las instituciones bancarias suministran, a través de sus productos y servicios, para crear fidelidad

y satisfacción en los segmentos de clientes seleccionados. Kaplan y Norton (2.002) indican que observaron un conjunto común de atributos, que organiza la propuesta de valor: 
Atributos de los productos y servicios: abarcan la funcionalidad del producto/servicio, su precio y calidad.

Relación con los clientes: incluye la entrega del producto/servicio al cliente, incluyendo la dimensión de la respuesta y plazo de entrega, y qué sensación tiene el cliente al utilizar la institución financiera.

Imagen y prestigio: refleja los factores intangibles que atraen a un cliente. La dimensión de imagen y prestigio permite a una institución financiera definirse a sí misma, de forma proactiva, para sus clientes.

Las instituciones financieras acostumbran a seleccionar dos conjuntos de medidas para su perspectiva del cliente. El primer conjunto, representa las medidas genéricas que todas las instituciones quieren utilizar (satisfacción del cliente, cuota de mercado y la retención del cliente). El segundo conjunto de medidas representa los inductores de la actuación de los resultados del cliente; y responden a la pregunta "¿qué es lo que la empresa ha de entregar a sus clientes para alcanzar un alto grado de satisfacción, retención, adquisición y, por último, cuota de mercado?" (Kaplan y Norton, 2.002).

\section{Metodologia.}

Este estudio se fundamentó en un enfoque cualitativo y cuantitativo, con un proceso deductivo, el alcance fue descriptivo ya que se detalla una realidad,

Para el desarrollo de la presente investigación se usó diferentes métodos como el teórico, al igual que el método analítico- sintético para la revisión de la literatura, en lo que tiene que ver a sus aspectos teóricos necesarios para el desarrollo de la presente investigación. También se usó el método dialéctico, inductivo y deductivo ya que mediante la aplicación de estos métodos se realizó el análisis del tema.

Para fundamentar teóricamente el presente trabajo se utilizó la investigación documental, además se aplicó la investigación de campo, con el objetivo de recabar información de los clientes del sistema financiero de Chimborazo. Luego a partir de datos relacionados con otros hallazgos se desarrollan unas generalizaciones (inducción) que, a su vez, guardan relación con otras teorías ya existentes (explicación). Se desarrolló esta investigación, para conocer la realidad, a través de encuestas con la aplicación de cuestionarios para conocer la situación actual.

Para el cálculo de la muestra se consideró la población de 587.661 clientes del Sistema financiero de la provincia de Chimborazo en el año 2018, y mediante la aplicación de la formula se obtuvo una muestra de 384 encuestas a ser aplicadas. 


\section{Resultados}

El Sistema Financiero Ecuatoriano se compone por entidades bancarias privadas, banca pública, mutualistas y cooperativas de ahorro y crédito. Estas instituciones buscan canalizar el ahorro hacia la promoción del desarrollo económico y productivo de Ecuador. Por lo expuesto el estudio incluye a todas las entidades mencionadas anteriormente.

\section{Cuantificación de captaciones por parte del sistema financiero de la provincia de} Chimborazo

Tabla 1. Captaciones Chimborazo 2018

\begin{tabular}{|c|c|c|}
\hline ENTIDAD & CAPTACIONES & No. CLIENTES \\
\hline Bancos & $\$ 345.200 .000,00$ & 201.517 \\
\hline Cooperativas & $\$ 430.466 .926,80$ & 377.653 \\
\hline \begin{tabular}{l|l|} 
& Segmento 1 \\
\end{tabular} & $\$ 366.075 .361,15$ & 262.941 \\
\hline Segmento 2 & $\$ 40.084 .891,01$ & 52.582 \\
\hline Segmento 3 & $\$ 24.306 .674,64$ & 62.130 \\
\hline Mutualistas & $\$ 15.661 .738,97$ & 8.491 \\
\hline Total captaciones & $\$ 791.328 .665,77$ & 587.661 \\
\hline
\end{tabular}

Fuente: La banca en Ecuador un enfoque provincial ASOBANCA, Boletín captaciones SEPS Elaborado por: Los autores

Entre los resultados alcanzados, se estableció que sistema financiero de la provincia de Chimborazo recibió captaciones por un monto de \$791`328.665,77 dólares, de los cuales el $64,26 \%$ captaron las cooperativas, el 34,29\% los bancos, y tan solo el 1,44\% las mutualistas. Por lo expuesto, se concluye que las cooperativas son las entidades financieras que mayor participación de mercado tienen en la provincia de Chimborazo.

\section{Resultados perspectiva del cliente}

Una vez tabulados los resultados de las preguntas realizadas mediante la encuesta aplicada a los clientes del Sistema financiero en la provincia de Chimborazo, se obtuvo los siguientes resultados:

Pregunta 1. ¿Es cliente de alguna entidad financiera actualmente? 


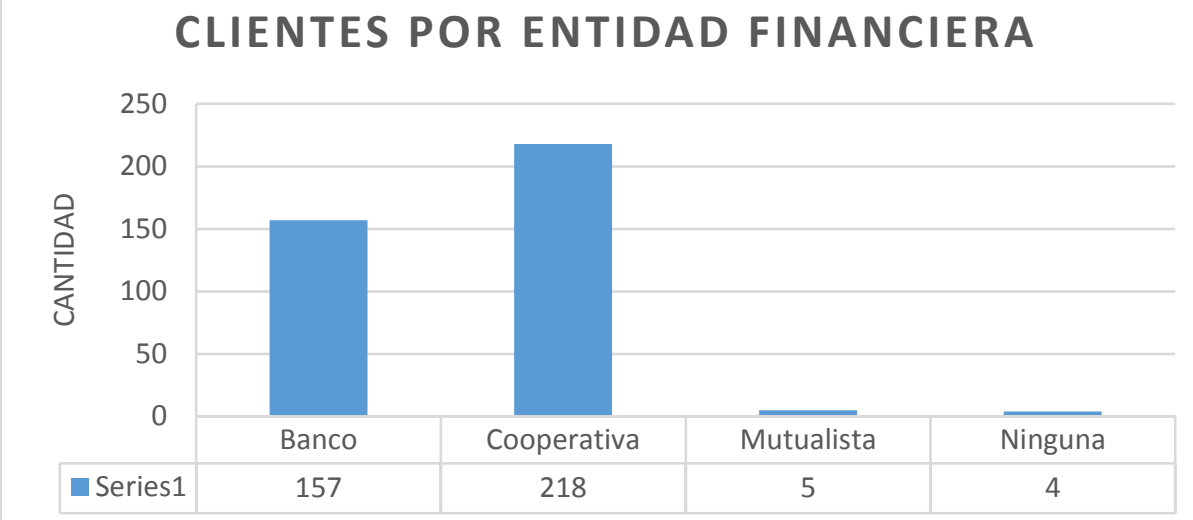

Gráfico 1. Clientes por entidad financiera

Fuente: Elaboración propia

Interpretación: En la encuesta realizada podemos verificar que la mayoría de las personas encuestadas el 56,77\% son clientes de cooperativas, el 40,89\% son clientes de bancos; el $1,3 \%$ son clientes de mutualistas, y el $1,04 \%$ no trabajan con el sistema financiero actualmente. Lo expuesto evidencia que las cooperativas de ahorro y crédito tienen la mayor participación de mercado en la Provincia de Chimborazo.

Pregunta 2. ¿Qué le motivo a ser cliente de una entidad financiera?

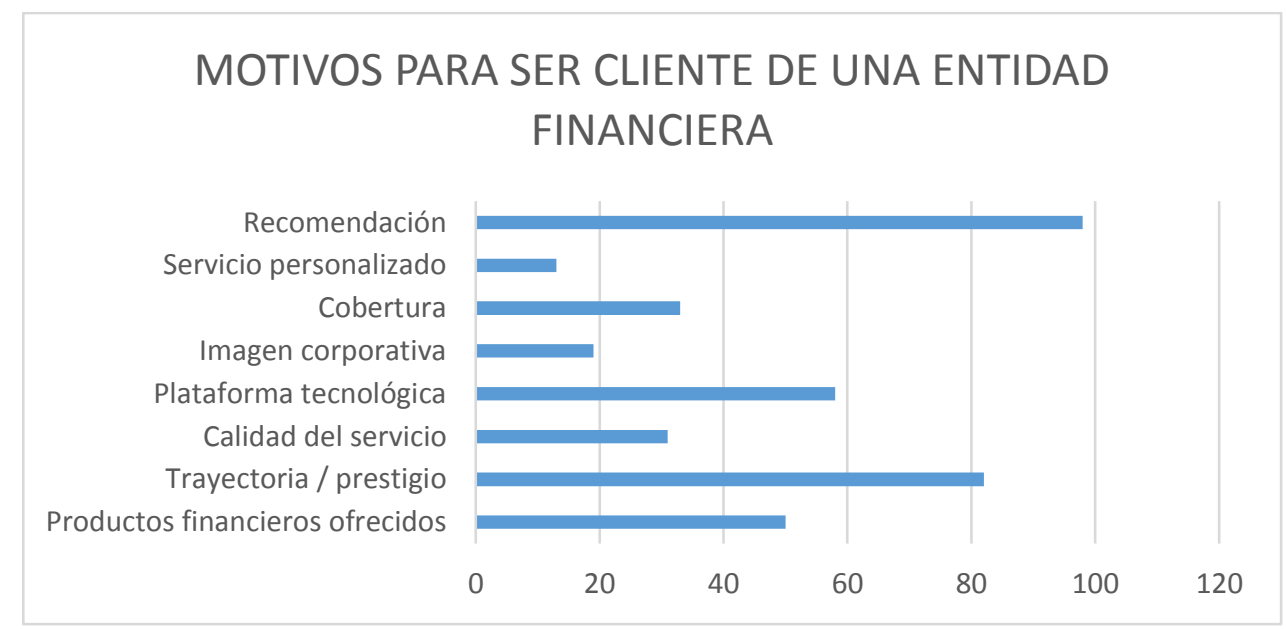

Gráfico 2. Motivos para ser cliente de una entidad financiera

Fuente: Elaboración propia

Interpretación: En esta pregunta se puede evidenciar que el $26 \%$ de los clientes acuden a una institución financiera por recomendación de otros clientes, el $21 \%$ por trayectoria y prestigio, el $15 \%$ por la plataforma tecnológica, el $13 \%$ por los productos financieros ofrecidos, el $9 \%$ por cobertura y disponibilidad de agencias, el $8 \%$ por calidad del servicio, el $5 \%$ por imagen corporativa y el $3 \%$ por el servicio personalizado. 
Pregunta 3. ¿Qué tan satisfecho está con el servicio o producto financiero que brinda su institución financiera actual?

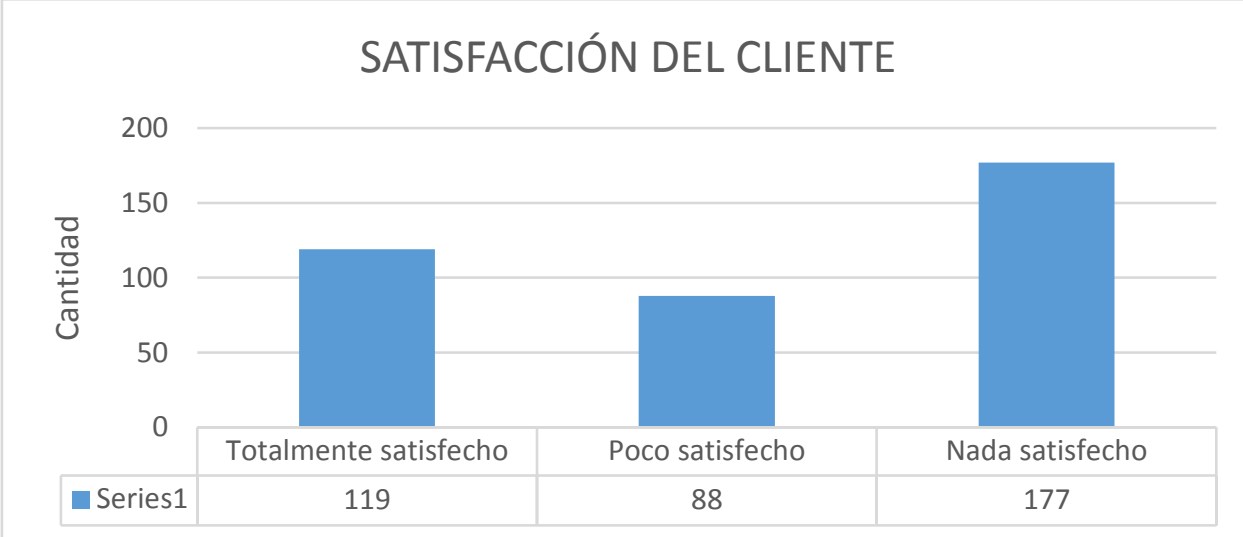

Gráfico 3. Satisfacción del Cliente

Fuente: Elaboración propia

Interpretación: En esta pregunta tenemos que el $46 \%$ de las personas encuestadas no están satisfechos con el servicio o producto que brinda la institución financiera, el $31 \%$ están totalmente satisfechos y el $23 \%$ están poco satisfechos. Con este antecedente es importante que las instituciones financieras desarrollen estrategias para mejorar la percepción por parte del cliente, y a la vez se logre la fidelización del mismo.

Pregunta 4. ¿Qué tipo de servicio o producto utiliza en la institución financiera?

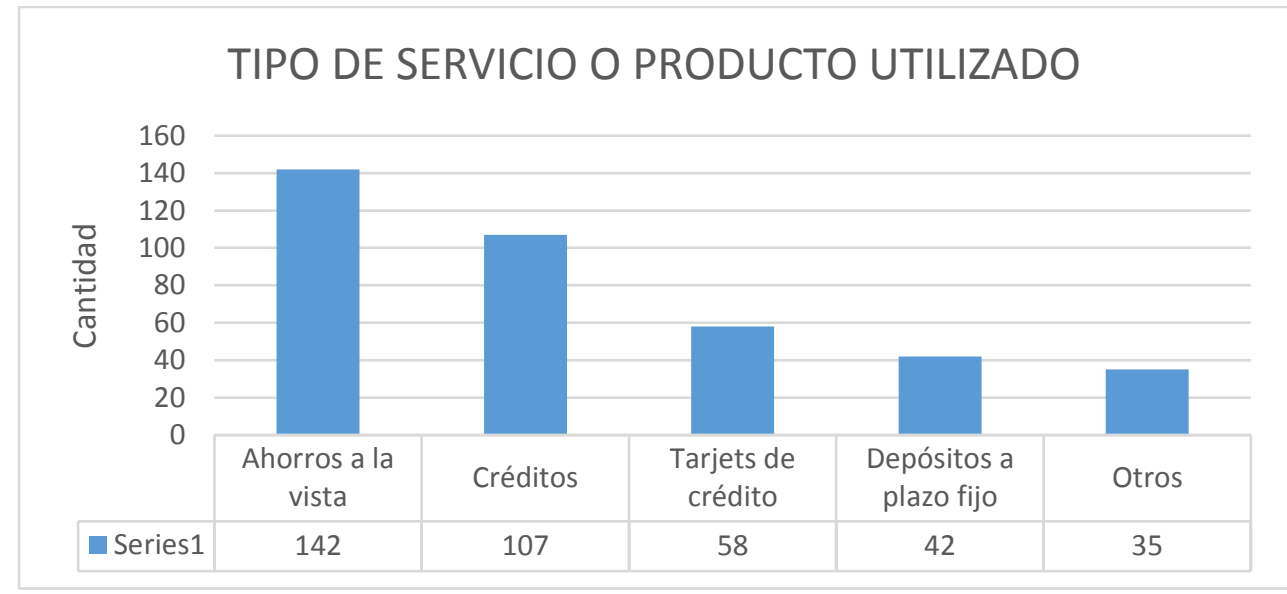

Gráfico 4. Tipo de servicio o producto utilizado

Fuente: Elaboración propia

Interpretación: Los resultados en esta pregunta permiten evidenciar que el servicio o producto más utilizado por los clientes del sistema financiero son los ahorros a la vista con el $37 \%$; el $28 \%$ créditos, el $15 \%$ tarjetas de crédito, el $11 \%$ depósitos a plazo fijo y el $9 \%$ otros servicios bancarios. 
Pregunta 5. ¿Qué aspectos considera usted para realizar inversiones en una entidad financiera?

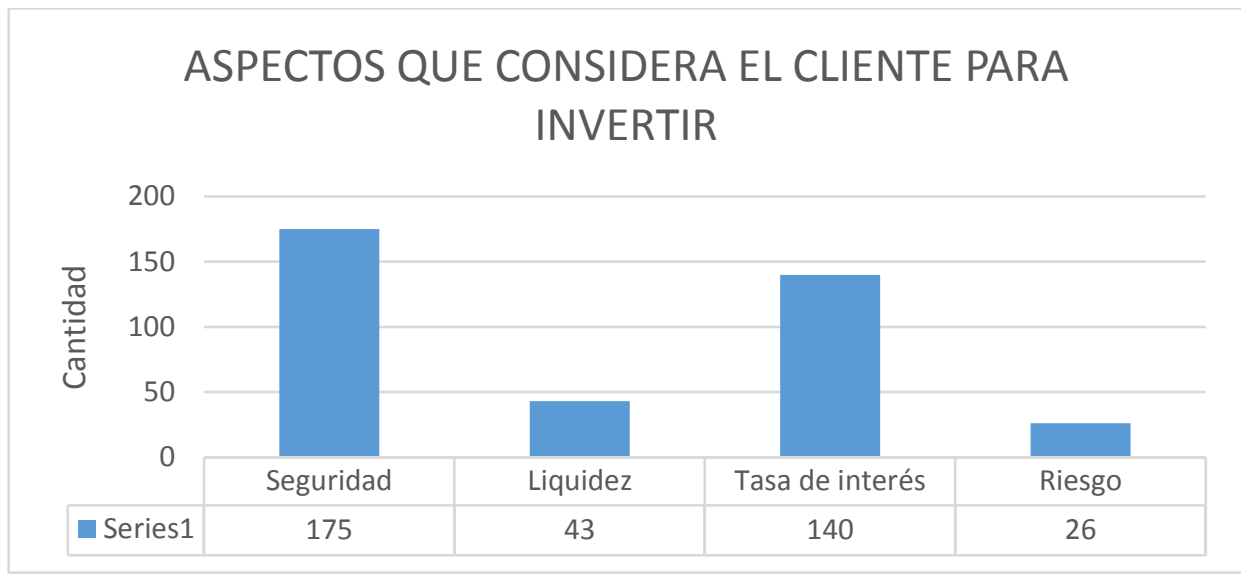

Gráfico 5. Aspectos que considera el cliente para invertir

Fuente: Elaboración propia

Interpretación: En esta pregunta se puede evidenciar que el 46\% de los clientes para invertir en una institución financiera, consideran la seguridad que le ofrece la institución, el 36\% las tasas de interés, el $11 \%$ la liquidez, y el $7 \%$ el riesgo. Por lo expuesto las instituciones financieras deben emprender en campañas de fortalecimiento institucional y de comunicación sobre productos, servicios y tasas de interés a los clientes.

Pregunta 6. ¿Conoce las tasas de interés que oferta el mercado financiero local?

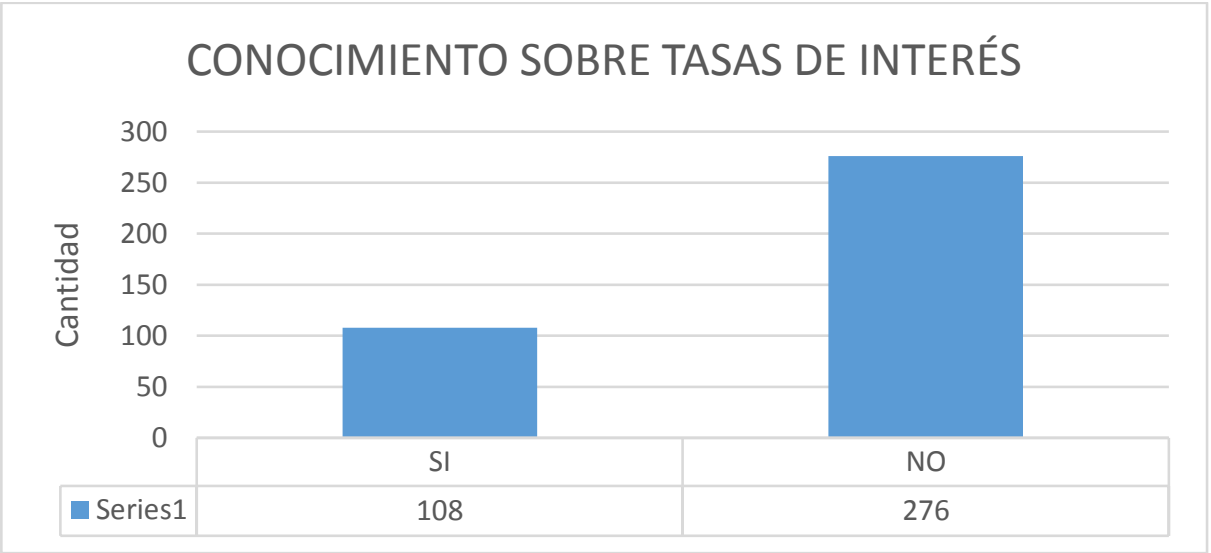

Gráfico 6. Conocimiento sobre tasas de interés

Fuente: Elaboración propia

Interpretación: Una vez analizados los resultados de la investigación en lo que corresponde al conocimiento de las tasas de interés, el $28 \%$ si conoce y el $72 \%$ desconoce. Bajo esta perspectiva es necesario que las instituciones financieras consideren generar información con 
respecto a las tasas de interés y de esta manera los clientes puedan acceder a los distintos productos que ofrecen.

\section{Propuesta de estrategias de cambio en captaciones}

Estrategia $N^{\circ} 1$ : Incrementar los depósitos a plazo Fijo

- Otorgar tasas pasivas competitivas.

- Identificar la necesidad de clientes de obtener un ingreso adicional por sus ahorros.

- Establecer metas de captación.

- Establecer políticas de incentivos dependiendo del tiempo y del monto (premiar el ahorro).

Estrategia $N^{\circ} 2$ : Fidelizar a los clientes actuales

- Mejorar la atención al cliente.

- Disponibilidad de tiempo dedicada por parte del agente de servicio al cliente

- Dominio y conocimiento de los servicios que se encuentra ofertando

- Brindar una adecuada bienvenida a los clientes.

- Crear un modelo de incentivos para los empleados.

- Incorporar un buzón de quejas.

- Establecer un plan de mejoras en función de las observaciones obtenidas.

\section{CONCLUSIONES}

- Las cooperativas de ahorro y crédito se han convertido en la mejor opción de inversión para los clientes del sistema finaciero de la provincia de Chimborazo, ya que manejan una tasa de interés muy alta con respecto a los bancos en sus depósitos a plazo fijo, gracias a esto en el año 2018 han captado el 64,26\% del mercado de captaciones.

- Según las encuetas realizadas a los clientes, tenemos que el $46 \%$ de las personas encuestadas no están satisfechos con el servicio o producto que brinda la institución financiera, el $31 \%$ están totalmente satisfechos y el $23 \%$ están poco satisfechos. Con este antecedente es importante que las instituciones financieras desarrollen estrategias para mejorar la percepción por parte del cliente, y a la vez se logre su fidelización.

- Según la perspectiva de los clientes, se pudo evidenciar que los aspectos más importantes que consideran para realizar una inversión son la seguridad de la entidad financiera y las tasas de interés en un $46 \%$ y $36 \%$ respectivamente, por lo expuesto las instituciones financieras deben emprender en campañas de fortalecimiento 
institucional y de comunicación sobre productos, servicios y tasas de interés a los clientes.

\section{REFERENCIAS BIBLIOGRÁFICAS}

Aguirre, D., \& Andrango, S. (2011). Preferencias en el uso de productos y servicios financieros que ofrecen las. Recuperado el 11 de 05 de 2019, de http://dspace.utpl.edu.ec/bitstream/123456789/3019/1/Aguirre\%20Gudino\%20Diana\%20 Alexandra\%20\%26\%20Andrango\%20Anrango\%20Susana\%20del\%20Rocio.pdf

Alexander, G., Sharpe, W., \& Bailey, J. (2003). Fundamentals of Investments. Pearson Education.

Avila, G. (2011). Asociaciones mutualistas. Recuperado el 12 de 05 de 2019, de https://es.calameo.com/books/00079367893a879b110be

Banco Central del Ecuador. (2009). Normas de creación. Recuperado el 06 de 05 de 2019, de https://www.bce.fin.ec/index.php/normas-de-creacion

Calderón, G. (15 de 09 de 2006). La misteriosa banca pública ecuatoriana. Recuperado el 13 de 05 de 2019, de https://www.elcato.org/la-misteriosa-banca-publica-ecuatoriana

Caraballo, A. (2011). Analisis del entorno de las cooperativas asociadas a CONFECOOP Caribe. Recuperado el 12 de 05 de 2019, de http://www.eumed.net/librosgratis/2013a/1314/cooperativa.html

Fuenmayor, B. (18 de 06 de 2018). ¿Depositar tus ahorros en bancos o cooperativas de ahorro? Recuperado el 06 de 05 de 2019, de https://produbanco.tusfinanzas.ec/category/sistemafinanciero/

Gitman, L., \& Joehnk, M. (2009). Fundamentos de inversiones. México: Pearson Educación.

Hernández, A., Hernández, A., \& Hernández, A. (2005). Formulación y evaluación de proyectos de inversión. México: S.A. Ediciones Paraninfo.

Heyman, T. (2001). Inversión en la globalización : análisis y administración de las nuevas inversiones mexicanas. México: Milenio.

Oppers, E. (21 de 10 de 2011). Obtenido de https://blog-dialogoafondo.imf.org/?p=1302

RAMIREZ ARANGO, c. y. (2001). Obtenido de http://dspace.ucuenca.edu.ec/bitstream/123456789/1359/1/tcon432.pdf 
Recalde, D. (2010). Sistema financiero ecuatoriano. Recuperado el 07 de 05 de 2019, de https://www.academia.edu/7277427/SISTEMA_FINANCIERO_ECUATORIANO_I._BANCA_P RIVADA

Robayo, E. (2012). Sistema financiero. Recuperado el 26 de 03 de 2019, de https://www.academia.edu/10290750/SISTEMA_FINANCIERO_INTRODUCCION_EI_Sistem a

Roca , R. (2002). La tasa de interés y sus principales determinantes. Obtenido de http://economia.unmsm.edu.pe/org/arch_iie/arch_invest/doc_inv_DI-02-003.pdf

Roca, R. (10 de 2002). Obtenido de http://economia.unmsm.edu.pe/org/arch_iie/arch_invest/doc_inv_DI-02-003.pdf

Román, C. (2018). El crédito como aporte para el desarrollo de las actividades productivas, en la provincia de EI Oro, caso; Banco Delbank y solidario. Periodo 2005 al 2016. Recuperado el 02 de 05 de 2019, de http://dspace.utpl.edu.ec/bitstream/20.500.11962/22591/1/Rom\%C3\%A1n\%20L\%C3\%B3 pez\%2C\%20Cristhian\%20Vinicio.pdf

Romero, B. (2015). Tus Finanzas. Obtenido de https://tusfinanzas.ec/category/sistema-financiero/

Santander, B. (s.f.). Obtenido de http://www.santander.com/csgs/Satellite/CFWCSancomQP01/es_ES/Santander/Accionistas/Mexico/Otra-informacion-de-interes/Glosario?cidSel=1278678993260

Superintendencia de Bancos. (2010). Glosario de Términos. Recuperado el 12 de 05 de 2019, de https://www.superbancos.gob.ec/bancos/glosario-de-terminos/

vanessa, R. F., \& milton, T. R. (2005). Obtenido de https://www.dspace.espol.edu.ec/bitstream/123456789/15877/3/Resumen\%20para\%20C ICYT.pdf

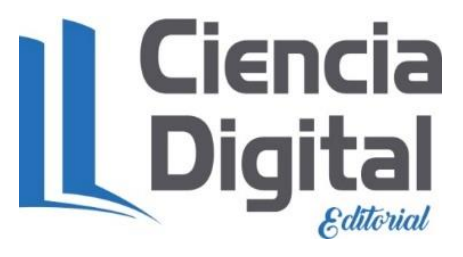




\section{PARA CITAR EL ARTÍCULO INDEXADO.}

Pazmiño Garzón, D., Moncayo Sánchez, Y., Tapia Bonifaz, A., \& Jarrin Zambrano, G. (2019). La captación de inversiones por el sistema financiero de Chimborazo, análisis desde la perspectiva del cliente. Ciencia Digital, 3(3.1), 353-374. https://doi.org/10.33262/cienciadigital.v3i3.1.708

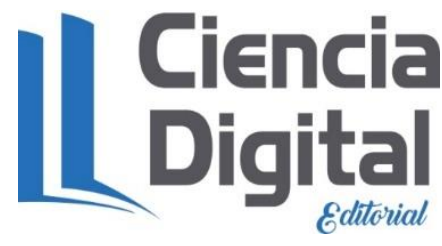

El artículo que se publica es de exclusiva responsabilidad de los autores y no necesariamente reflejan el pensamiento de la Revista Ciencia Digital.

El artículo queda en propiedad de la revista y, por tanto, su publicación parcial y/o total en otro medio tiene que ser autorizado por el director de la Revista Ciencia Digital.
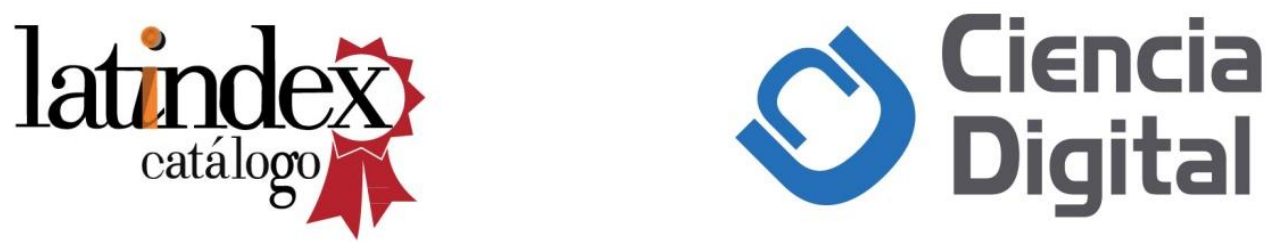